\title{
Perfiles de adolescentes on line y su comportamiento en el medio interactivo
}

\section{Online teen profiles and their behavior in the interactive media}

\author{
Belinda de Frutos Torres \\ Profesora Contratada Doctora (Facultad de CC. Sociales, Jurídicas y de la
} Comunicación, Universidad de Valladolid, Campus de Segovia)

\section{María Sánchez Valle}

Profesora Adjunta (Departamento de Comunicación Audiovisual y Publicidad, Universidad CEU San Pablo)

\section{Tamara Vázquez Barrio}

Profesora Colabradora Doctora (Departamento de Periodismo, Universidad CEU San Pablo)

Fecha de recepción: 2 de abril de 2012

Fecha de revisión: 15 de febrero de 2013

Para citar este artículo: Frutos Torres, B., Sánchez Valle, M. y Vázquez Barrio, T. (2014): Perfiles de adolescentes on line y su comportamiento en el medio interactivo, Icono 14, volumen (12), pp. 374-397. doi: 10.7195/ ri14.v12i1.208 


\section{Resumen}

Internet se ha convertido en el medio de referencia entre los jóvenes $y$, aunque su uso está muy extendido, se pueden identificar perfiles de usuarios respecto a la actitud hacia el medio interactivo y su estilo de navegación. El objetivo del estudio se centra en identificar dichos perfiles y ponerlos en relación con su comportamiento en Internet. El estudio utiliza una metodología correlacional, con una muestra de conveniencia formada por 320 jóvenes entre 14 y 19 años, procedentes de varios centros de educación secundaria. Los resultados muestran que se pueden identificar varios patrones de adolescentes en el consumo de medios interactivos en función de su estilo de navegación y actitud hacia el medio. El estilo de navegación exploratorio está asociado a participar en actividades comerciales de la red y a dedicar más tiempo al medio interactivo, particularmente de lunes a viernes. Así mismo, los jóvenes que se enfrentan al medio con una actitud más confiada, y ven el medio como un vehículo de socialización y éxito social, también dedican más tiempo al medio interactivo y participan en mayor medida de las propuestas publicitarias que se ofrecen en Internet.

\section{Palabras clave}

Perfiles de uso de Internet - Publicidad en Internet - Jóvenes y adolescentes - Comportamiento en Internet.

\section{Abstract}

Internet has become the reference medium among young people and although its use is widespread can be identified user differences on the attitude towards the interactive media and different navigating styles. The aim of the study focuses on identifying user's profiles and test the relationship with media consumption pattern and the online behavior. The study is based on a correlational methodology, carried out with a convenience sample consisting of 320 young people between 14 and 19 years from various secondary schools. The results identify several patterns of adolescents in interactive media consumption based on their attitude toward the media and their navigation pattern. The exploratory navigation style is associated

ICONO14 | Año 2014 Volumen $12 N^{\circ} 1$ | ISSN: 1697-8293 | DOI: ri14.v12i1.208 
with greater participation in commercial activities on Internet and more time spend on the media particularly during the working days. Likewise, young people who face the media with a more confident attitude and see the medium as a vehicle of socialization and social success also devote more time connected to interactive media and is more likely to respond to the advertising proposals offered online.

\section{Key Words}

Internet profiles - Internet advertising - Adolescents and youth - Online behavior

\section{Introducción}

Los medios interactivos están cambiando el panorama mediático en cuanto al hábito de consumo se refiere, particularmente en el ámbito de los más jóvenes, que han incorporado las nuevas tecnologías con mayor facilidad e intensidad que otros segmentos de la población. Según los datos del último estudio de Equipamiento y Uso de Tecnologías de la Información y la Comunicación en el Hogar el 91,5\% de los jóvenes entre 16 y 25 años ha accedido a Internet al menos una vez por semana, este porcentaje es algo más bajo para los niños de 12 a 15 años situándose en el $87,5 \%$ (INE, 2011). Los datos recogidos por la última ola del Estudio General de Medios apuntan en este mismo sentido al situar la penetración del medio interactivo entre los jóvenes de 14 y 19 años en el 74,4\% (AIMC, 2012) ${ }^{1}$.

Varios trabajos de investigación e informes sectoriales recientes han profundizado en el uso que los menores en España hacen de los medios interactivos (ver recientes trabajos de revisión publicados por Peréz-Latre, Portilla y Sánchez Blanco, 2011 o el de García Jiménez, López de Ayala, y Gaona Pisonero, 2011). De ellos se infiere que el medio interactivo ofrece un amplio abanico de posibilidades en términos de entretenimiento, información, comunicación, participación y consumo. La mayor parte de las publicaciones recogen una profusa descripción de los usos y actividades que los menores llevan a cabo en la red, siendo los adolescentes y los jóvenes un público prioritario que centra su interés (Bringué y Sádaba, 2011; Garmendia, Garitaonandia, Martínez y Casado, 2011; Monge y Olabarri, 2011; Sánchez y Frutos, 2010; Sánchez-Martínez y Otero Puime, 2010; Sánchez Burón y Fernández Martín, 2010). 
A medida que el uso del medio interactivo va madurando emergen otros matices que se ponen de manifiesto en diferentes patrones de consumo. Podríamos plantear que no todos los jóvenes realizan las mismas cosas en Internet. En algunos trabajos realizados con adolescentes se ha tomado el tiempo dedicado como variable de referencia para identificar tipologías de usuarios. Así, en el reciente trabajo realizado con jóvenes Sánchez-Martínez y Otero Puime (2010) distinguen entre un "uso muy alto de Internet" (más de 5 horas al día conectado incluido los fines de semana), "uso alto" (4 horas de conexión entre semana o 5 horas en fines de semana), "uso medio" (si se conecta entre 1 y 3 horas al día durante la semana) y "uso bajo" (si se conecta menos de 1 hora al día durante la semana o menos de 3 horas durante el fin de semana). En otro ámbito de estudio, Bringué y Sádaba (2011) clasifican a los menores según su uso de las redes sociales, categorizándole en "usuarios avanzados" (tienen más de un perfil en redes sociales), "usuarios" (tienen un perfil en redes sociales) y "no usuarios". En ambos trabajos se evidencia el comportamiento diferenciado de los menores según este criterio de clasificación respecto a variables sociodemograficas como la edad y el género o bien su asociación con otros aspectos como el rendimiento académico o la relación con los padres.

Otra fuente de diferencias podría tener una base generacional. Así el término "generación digital" o "nativos digitales", adoptado hace ya más de una década, refleja el alcance que tiene la experiencia interactiva como modo de entender la vida para una generación pionera por su experiencia. Los nativos digitales se diferencian de los inmigrantes digitales por su modo de acceder y procesar la información, donde el medio interactivo se ha convertido en su principal vehículo de relación, de expresión y de identidad (Rubio, 2010).

Por otra parte, la investigación sobre el comportamiento de los usuarios en el medio interactivo ha puesto de manifiesto diferencias dependiendo de su experiencia en el medio (Muylle, Moenaert y Despotin 1999; Dahlen, Rash y Rosengren, 2003; Assael, 2005). Yang (2005) a propósito del comportamiento en Internet identificó patrones de navegación en la red diferenciados según la situación y el estilo del internauta. En este trabajo se recogen tipologías que van desde surfear de forma exploratoria sin tener muy claro una meta a otro tipo de navegación por objetivos y medios específicos donde el usuario sabe qué busca y dónde encontrar- 
lo. La pertenencia a uno u otro perfil vendría explicada por múltiples factores de tipo personal, actitudinal e incluso situacional. Siguiendo con este planteamiento parece interesante explorar patrones más cercanos a variables psicográficas y actitudinales que den cuenta del uso diferenciado del medio. En esta línea Schiffman, Sherman y Long (2003) en una investigación realizada con población general, pusieron en relación un conjunto de actividades desarrolladas en la web con la identificación de valores personales generales asociados a Internet (i.e. realización personal, logro, diversión y disfrute de la vida, emoción y respeto). Otra investigación de Assael (2005) plantea igualmente la necesidad de categorizar los usuarios según su comportamiento en la red buscando variables más cercanas a perfiles psicológicos y de estilo de vida. En su estudio categorizó a los usuarios en diferentes perfiles según su actividad preferente en la web (generalistas, bajar contenidos, desarrollo personal, entretenimiento, búsqueda de gangas y socializadores) y desarrolló un perfil de estilo de vida para cada uno.

Dentro de los estudios realizado con los menores y los medios interactivos uno de los objetivos se centra en los riesgos asociados al medio, una preocupación que comparten investigadores, educadores y padres. En España cabe mencionar el trabajo desarrollado por Garmendia y col. (2011), dentro de la red de trabajo EU Kids On line, que lidera la investigadora Sonia Livingstone en la que están implicados 33 países.

Dentro de las cuestiones relacionadas con la seguridad de los menores en la red, nuestro interés se centra en conocer hasta qué punto los menores son conscientes de los riesgos, o lo que es lo mismo, en qué medida muestran una actitud de confianza hacia Internet en general, y las redes sociales en particular. Los resultados publicados sobre este tema muestran que la preocupación de los padres o tutores por el uso que los menores hacen de la red es mayor en España que en el resto de países europeos, según informan Garitaonandia y Garmendia (2009), pero también somos un país donde se produce un bajo control parental.

Por otra parte, los estudios publicados indican que los menores tienden a tener un exceso de confianza sobre su manejo y control de Internet (Livingstone, 2010). El uso frecuente de una tecnología no implica que la domine, ni que con- 
trole completamente como pusieron de manifiesto Monge y Olabarri (2011) en un estudio con universitarios, y su conocimiento sobre las condiciones de privacidad de las redes sociales. En una reciente encuesta publicada por el Eurobarometer ${ }^{2}$ y desarrollada en el ámbito europeo se mostraba que la mayor parte de los menores afirma saber más de Internet que sus padres, pero su aprendizaje en el manejo de las redes sociales ha sido intuitivo. El $72 \%$ afirma que ha recibido ayuda de sus propios compañeros y el $44 \%$ manifiesta que la ayuda recibida sobre la seguridad en Internet ha venido de su grupo de amigos.

En un trabajo previo, realizado por el equipo investigador con grupos de discusión, se recogió que la seguridad y la privacidad en Internet formaba parte de las preocupaciones de los menores, sin embargo, su comportamiento en la red no era coherente con esta preocupación, que tampoco se generalizaba entre todos los menores (Frutos y Vázquez, 2012). El concepto de alfabetización digital, término tomado del media literacy, hace referencia a un amplio espectro de habilidades y competencias vinculadas al acceso, análisis, evaluación y creación de mensajes en un conjunto de entornos mediados. Una de las cuestiones más importantes del concepto es que no se centra sólo en las habilidades técnicas en el sentido de competencias instrumentales, también resalta la importancia de otras habilidades como la capacidad crítica, de análisis u otras habilidades creativas, aspectos que se habían obviado en las competencias en medios convencionales. La creación y producción de contenidos exige niveles de competencia mediática mucho mayor que el consumo de los mismos (Phang y Schaefer, 2009). Según Hasebrink, Livingstone y Haddon (2008) cuanto más tiempo están los menores en el medio interactivo y cuanto más usan determinados servicios, mayor será la probabilidad de desarrollar habilidades, asumiendo que éstas se desarrollan con el uso y con la edad. En este sentido, se ha podido comprobar a través de la metodología de los grupos de discusión que entre los menores se produce un proceso de autoregulación en lo que respecta a su uso de las redes sociales, después de un uso implosivo de determinadas aplicaciones este se modera con el paso del tiempo (Frutos y Vázquez, 2012).

Así pues, parece relevante que los menores tomen conciencia de la importancia de tener una actitud crítica a la hora de enfrentarse al medio interactivo. Deben ser conscientes de cuestiones que afectan a su privacidad, que son muy importantes a 
la hora de gestionar su imagen y su reputación actual y futura. Los menores también deben saber que detrás de las plataformas proveedoras de servicios gratuitos, de las que son partícipes las redes sociales, hay un interés comercial que se puede vestir de muchos modos y maneras.

El medio interactivo ha evolucionado muy rápidamente respecto a las acciones publicitarias, tanto en volumen de inversión, como en las modalidades bajo las que se presenta. Aunque la valoración que hacen los usuarios de la publicidad en este entorno no es muy favorable. Experiencias con formatos publicitarios intrusivos, el miedo a los virus, la ausencia de control y la publicidad engañosa, maliciosa ha propiciado la desconfianza o la molestia que se atribuye a la publicidad en Internet (Frutos y Gutiérrez, 2005; Frutos y Sánchez, 2011). A pesar de la desconfianza que genera el medio, las acciones publicitarias en Internet también consiguen una mayor implicación y participación de los usuarios. El medio interactivo trae consiguo mayor participación de los usuarios, que ha supuesto un cambio de paradigma en la comunicación mediada (Frutos, 2010). El papel más activo del usuario se traduce en una mayor implicación que influye positivamente en los efectos del mensaje publicitario (Frutos y Sánchez, 2006). Además las acciones comerciales en las redes sociales se perciben más positivamente que en los sitos o páginas web convencionales (The Cocktail Analysis, 2011). En este escenario surge la cuestión que se aborda en este trabajo sobre el modo en que los menores se enfrentan a las acciones comerciales y publicitaras en el entorno interactivo.

\section{Objetivos}

El objetivo del trabajo se centra en identificar perfiles de usuarios en el colectivo de adolescentes y jóvenes y ponerlo en relación con su comportamiento en el medio interactivo. En concreto, se definen cuatro perfiles de usuarios basados en el estilo de navegación y actitud hacia la red, En primer término, distinguimos entre estilo de navegación focalizado frente al exploratorio; en segundo lugar, consideramos la actitud de confianza versus escepticismo hacia las redes sociales. El primer objetivo del estudio se centra en contrastar empíricamente los perfiles identificados.

El segundo objetivo se ocupa de poner en relación los perfiles de los usuarios

DOI: ri14.v12i1.208 | ISSN: 1697-8293 | Año 2014 Volumen 12 Nº 1 | ICONO14 
con su comportamiento en Internet. Dentro de la diversidad de actividades que los jóvenes realizan en el medio interactivo se ha optado por medir su grado de implicación en la publicidad y las acciones comerciales en el medio. Así mismo se ha utilizado el tiempo de conexión en Internet como indicador global de la actividad de los menores en Internet.

\section{Metodología}

El estudio se ha realizado siguiendo una metodología correlacional. A través de la encuesta diseñada por el equipo de investigación, se recogieron las respuestas de los adolescentes y jóvenes mediante un cuestionario auto-cumplimentado. Se ha utilizado una muestra de conveniencia formada por 323 adolescentes, entre 14 y 19 años de edad, a los que se contactó a través de 6 centros de enseñanza media ubicados en Ciudad Real, Madrid, Málaga y Segovia. En la elección de los centros se realizó de modo que estuvieran representados centros públicos, concertados y privados. Los datos fueron recogidos entre abril y mayo de 2011.

\section{Medición de los estilos de navegación}

En el desarrollo de la investigación se ha creado una escala que tiene como objetivo medir el estilo de navegación libre en la red ${ }^{3}$. La primera dimensión está formada por 6 afirmaciones que reflejan diversas situaciones en las que el usuario tiene un comportamiento exploratorio de la red y está abierto a descubrir nuevos contenidos o bien un estilo directo. Frente a esta dimensión, se plantea otra forma de navegación dirigida o focalizada, donde el usuario suele seguir su propia rutina, se guía por los objetivos y no se deja llevar por otros recursos, que ha sido evaluada con 3 afirmaciones. Por otra parte, la escala mide la confianza que le ofrecen las redes sociales y cómo constituyen una herramienta social. La escala consta de 3 afirmaciones del estilo "Creo que gracias a las redes sociales puedes llegar a ser muy popular y tener mucho éxito social" o "Para mí la red social es una oportunidad para enterarme de muchas cosas y estar en contacto con otras personas". Por último, la cuarta dimensión recoge el escepticismo asociado a las redes sociales con cuestiones del tipo: "Soy bastante escéptico para participar en eventos u otras acciones en las redes sociales que pueden dejar visible mi perfil a desconocidos" 0 "Hoy en día no te puedes fiar mucho de los contenidos de las redes sociales porque

ICONO14 | Año 2014 Volumen $12 N^{\circ} 1$ | ISSN: 1697-8293 | DOI: ri14.v12i1.208 
la gente se aprovecha del anonimato y muchos contenidos no son ciertos".

\section{Medición de la implicación en actividades comerciales y publicitarias en la red}

En lo que respecta al comportamiento en Internet y las redes sociales se ha recogido la participación en un conjunto de 10 cuestiones vinculadas a experiencias publicitarias, comerciales o juegos patrocinados por un anunciante en Internet. El tipo de acciones recogidas van de menor a mayor grado de implicación. Por ejemplo: fijarse en el contenido de un anuncio publicitario o hacer click en el anuncio, entrarían dentro de las acciones con menor grado de implicación. Unirse a un evento, participar en un juego, concurso o similar en el que solicitaban datos personales o de registro reflejan un grado de participación más alto. Para tener una referencia general del grado de participación en este tipo de actividades se ha calculado una variable sumativa ${ }^{4}$ global a partir de la participación en cada una de las actividades indicadas.

Por último, se ha medido el tiempo de consumo diario del medio durante la semana (de lunes a viernes) y durante el fin de semana que se ha computado en horas de consumo al día.

\section{Resultados}

\subsection{Consumo del medio interactivo}

Los primeros resultados el grado de exposición al medio interactivo. El 87,3\% declara que se conecta Internet todos los días, el $12,4 \%$ se conecta de vez en cuando y sólo uno de los participantes afirma que nunca accede a la web fuera del centro de estudios. El tiempo medio de conexión a lo largo de un día de lunes a viernes es de 5 horas y 48 minutos (Desviación típica $=6,4$ ), durante el fin de semana la media aumenta hasta las 8 horas y 18 minutos (Desviación típica $=6,8$ ). La incorporación de los nuevos terminales Smartphone ${ }^{5}$ sin duda ha contribuido al tiempo de conexión del colectivo, según se ha podido constatar entre el 4,4\% y $7,9 \%$ de la muestra está permanentemente conectado a Internet, posiblemente debido a estos nuevos dispositivos.

DOI: ril4.v12i1.208 | ISSN: 1697-8293 | Año 2014 Volumen 12 N 1 | ICONO14 
Un examen de la distribución de frecuencias refleja que ésta toma cierto grado de asimetría negativa, es decir, hay mayor número de puntuaciones en los valores más bajos de la distribución. Tomando como referencia el tiempo de conexión, se ha clasificado a la muestra en cuatro grupos aproximadamente del mismo tamaño. El primero, denominado de "bajo consumo", reúne a un cuarto de los encuestados que en cualquier día de la semana está conectado menos de dos horas y durante el fin de semana menos de 3 horas y media. El segundo grupo, de "consumo moderado", se conecta durante la semana de 2 a 3 horas y media, mientras que el fin de semana la conexión se sitúa entre 3 horas y media y 7 horas. El tercer grupo, que se puede calificar de "consumo alto", está conectado durante la semana entre 3 horas y media y 7 horas, y en el fin de semana de 7 a 10 horas y media. El último grupo, denominado de "consumo muy alto", durante la semana se conecta más de 7 horas al día y durante el fin de semana se conecta más de 10 horas y media al día.

\subsection{Experiencia publicitaria en la red social}

En el cuadro $n^{0} 1$ se muestra el grado de participación en actividades publicitarias o relacionadas con una actividad comercial. Algo más de la mitad de los participantes en el estudio reconocen haber participado en algún juego ${ }^{6}$ que posteriormente ofrecen un servicio o detrás hay publicidad de algún anunciante. La segunda acción más señalada ha sido fijarse en algún contenido publicitario porque despertaba su interés, señalado por el 53\%. La tercera acción referida con más frecuencia ha sido hacerse fan de una marca a través de las redes sociales, señalada por uno de cada tres jóvenes ${ }^{7}$. Este tipo de acciones, que se han introducido a la par que el desarrollo de las propias redes sociales, recoge un tipo de implicación mucho más alta desde el punto de vista publicitario, puesto que es el propio usuario el que inicia la relación o la interacción con el anunciante. En este sentido apunta la siguiente cuestión que recoge la proporción de usuarios que han hecho clic en un anuncio voluntariamente, porcentaje que se sitúa en el $28,6 \%$. Algo por debajo está la participación en eventos 0 acciones similares promovidas por una marca o un anunciante en la red social, que se sitúa en el $26,2 \%$. Cuando la acción tiene una contrapartida en forma de premios, descuentos, regalos o similares la participación se ha 
situado aproximadamente en el $25 \%$. Un porcentaje semejante ha reconocido preguntar en su red social antes de realizar una compra o utilizar los servicios de un anunciante. En los casos en los que la participación exige el registro a partir de los datos personales, la implicación es aún mayor, puesto que estos datos pueden ser utilizados posteriormente con fines comerciales. Los resultados ponen de manifiesto que sólo el $19 \%$ ha dado alguna vez sus datos personales a cambio de una recompensa de estas características. Las dos últimas cuestiones reflejan acciones relacionados con marcas pero, a diferencia de las anteriores, la acción no viene por iniciativa del anunciante sino que recae en la voluntad del usuario. Por una parte, se recoge si los usuarios han iniciado eventos o acciones similares en contra de alguna marca comercial en la red social, cuestión que ha confirmado el 17,6\%. Y por último, el $12,6 \%$ ha hecho comentarios en el perfil de una marca comercial.

El grado de implicación en las actividades publicitarias y comerciales se ha computado teniendo en cuenta las respuestas a los 10 indicadores descritos anteriormente. Los resultados globales muestran que esta variable tiene una puntuación media 2,9 (Desviación típica=2,2). Por lo tanto, podemos considerar que en conjunto los adolescentes han participado en 3 actividades de este tipo. Solo el $11,9 \%$ no ha participado en ninguna de las actividades recogidas y sólo el $13,7 \%$ ha señalado más de cuatro actividades.

En conjunto, estos resultados son relativamente relevantes puesto que varios estudios previos habían planteado reiteradamente la percepción negativa de la publicidad durante la navegación. Esta aparente contradicción se puede interpretar de diversos modos, una posible explicación es que se ha sobregeneralizado cuando se habla del rechazo hacia la publicidad en el medio interactivo. Cuando la publicidad es coherente con los intereses del receptor este se puede llegar a involucrar con más intensidad que en un medio convencional, lo que pone de manifiesto el cambio de paradigma en la comunicación interactiva. 


\section{Tabla $n^{\circ} 1$. Participación en actividades relacionadas con la publicidad $u$ otras acciones publicitarias en Internet}

\begin{tabular}{|l|c|c|}
\hline Actividades & No (\% fila) & Sí (\% fila) \\
\hline $\begin{array}{l}\text { ¿Has participado en pequeños juegos (dar al ratón, preguntas sobre } \\
\text { el test de inteligencia, o similares ...) que luego ofrecen un servicio } \\
\text { o publicidad? }\end{array}$ & 42,9 & 57,1 \\
\hline $\begin{array}{l}\text { ¿Te has fijado en algún anuncio cuando estabas navegando dentro } \\
\text { de una página de Internet porque te interesaba? }\end{array}$ & 47,0 & 53,0 \\
\hline ¿Te has hecho fan de alguna marca en tu red social? & 67,0 & 33,0 \\
\hline $\begin{array}{l}\text { ¿Has hecho clic sobre un anuncio (tipo banner) cuando estabas } \\
\text { navegando porque te ha llamado la atención (por su originalidad, } \\
\text { porque era diferente, ..) }\end{array}$ & 71,4 & 28,6 \\
\hline $\begin{array}{l}\text { ¿Te has unido a algún evento o acción promovido por una marca en } \\
\text { tu red social? }\end{array}$ & 73,8 & 26,2 \\
\hline $\begin{array}{l}\text { ¿Has participado alguna vez en concurso o promociones para obte- } \\
\text { ner un premio, descuentos o similares? }\end{array}$ & 75,3 & 24,7 \\
\hline $\begin{array}{l}\text { ¿Has preguntado por un producto/ marca en tu red social antes de } \\
\text { realizar una compra o tomar una decisión sobre un producto? }\end{array}$ & 75,5 & 24,5 \\
\hline $\begin{array}{l}\text { ¿Has participado en juegos desarrollados por una marca conocida } \\
\text { que te pedían registrarte? }\end{array}$ & 81,2 & 18,8 \\
\hline $\begin{array}{l}\text { ¿Te has unido a algún evento o grupo en contra de alguna marca en } \\
\text { tu red social? }\end{array}$ & 82,4 & 17,6 \\
\hline $\begin{array}{l}\text { ¿Has hecho comentarios en el muro de una marca comercial desde } \\
\text { tu red social? }\end{array}$ & 87,4 & 12,6 \\
\hline
\end{tabular}

Fuente: Elaboración propia

\subsection{Perfil de usuarios}

Para la identificación de los perfiles se llevó a cabo un análisis factorial componentes principales. La prueba de adecuación muestral de Kaiser-Meyer-0lkin es igual a 0.683 , lo que indica su adecuación para la aplicación del análisis de varianza. La prueba de esfericidad de Bartlett rechaza la hipótesis de ausencia de relación entre las variables, por lo tanto, podemos considerar que la matriz de datos es apta para llevar a cabo un análisis factorial. En el análisis se extrajeron 4 dimensiones que explican el $42,5 \%$ de la varianza. En el cuadro $n^{0} 2$ se muestra la matriz de

ICONO14 | Año 2014 Volumen $12 N^{\circ} 1$ | ISSN: 1697-8293 | DOI: ri14.v12i1.208 
pesos de cada ítem sobre cada dimensión después de aplicar la rotación oblimin para su mejor interpretación. La descripción de las dimensiones muestra de forma bastante clara la configuración de cuatro estilos en relación con la experiencia en el medio interactivo. La dimensión que emerge de forma más evidente y que da cuenta de mayor variabilidad es la dimensión de "confianza en la red". El factor reúne cinco ítems con pesos por encima de 0,40 y está asociada con un perfil de socialización a través de la red y un sentimiento de confianza en el medio. Afirmaciones del tipo: "me gusta buscar personas desconocidas con intereses similares a los míos...", o "creo que gracias a las redes sociales puedo llegar a ser muy popular y tener mucho éxito", o la afirmación que tiene un peso negativo "hoy en día no te puedes fiar mucho de los contenidos de las redes sociales (...)". La segunda dimensión recoge la faceta de navegación con un "estilo explorador", en el sentido de búsqueda de novedades y sensaciones. Seis de las preguntas propuestas saturan en esta dimensión entre las que destacan afirmaciones como: "suelo encontrar contenidos casualmente pasando de un contenido a otro", "cuando encuentro una página que me interesa lo agrego a mis favoritos", "confío en comentarios de otros usuarios cuando voy a realizar una compra", "buscar en internet es una forma de entretenimiento". La tercera dimensión está definida por el estilo de "navegación focalizado", en el que se realiza un uso funcional del medio, las búsquedas están muy dirigidas, e incluso la actividad en las redes sociales está dirigida a las relaciones más cercanas e intimas. Afirmaciones del tipo: "tengo una rutina muy definida cuando navego en internet y no suelo entrar en sitios que no conozco..", "cuando hago búsquedas en la red (...) voy directamente a lo que me interesa y no suelo explorar por curiosear". La cuarta dimensión se puede definir en torno al "escepticismo hacia las redes sociales" donde encontramos afirmaciones como: "pienso que en Internet hay contenidos que valen la pena pero también hay mucha basura", "soy bastante crítico con los contenidos que veo en las redes sociales..." o "soy bastante escéptico en participar en eventos u otras acciones en las redes sociales".

Por lo tanto, se ha comprobado empíricamente que los jóvenes responden a cuatro perfiles que giran en torno a los usos diferenciados en la red. En primer término la red se percibe como un vehículo de socialización hacia el que se muestra una gran confianza (dimensión 1), en segundo lugar, se aborda la red desde un estilo explorador como fuente de información y estimulación (dimensión 2). La tercera 
dimensión viene definida por un uso focalizado de la red, en este sentido el perfil encaja en una tipología más funcional en la que los usuarios utilizan la red para satisfacer intereses muy concretos (dimensión 3). La última faceta de los perfiles viene determinada por el escepticismo hacia las redes sociales, donde los jóvenes se muestran críticos con los contenidos, conscientes de la privacidad y que en general mantienen una actitud preventiva hacia el medio interactivo (dimensión 4).

Se ha comparado la puntuación factorial obtenida en cada uno de los perfiles (método de regresión) según la variable género y no ha mostrado diferencias estadísticamente significativas, esto es, ni los hombres, ni las mujeres obtienen una puntuación más alta en ninguno de los cuatro perfiles.

\section{Tabla $n^{\circ}$ 2: Resultados del Análisis factorial de componentes principales rotación oblimin sobre los estilos de navegación y perfiles actitudinales}

\begin{tabular}{|l|c|c|c|c|}
\hline & \multicolumn{3}{|c|}{ Dimensiones } \\
\hline & $\mathbf{1}$ & $\mathbf{2}$ & $\mathbf{3}$ & $\mathbf{4}$ \\
\hline $\begin{array}{l}\text { Me gusta buscar personas desconocidas con intereses similares } \\
\text { a los míos o simplemente curioseo en sus perfiles }\end{array}$ &, 698 &, 140 &, 024 &,- 108 \\
\hline $\begin{array}{l}\text { Me gusta explorar por páginas de viajes, subastas o similares } \\
\text { aunque no tenga intención de comprar }\end{array}$ &, 596 &,- 055 &,- 190 &, 302 \\
\hline $\begin{array}{l}\text { Navegando por la red descubro contenidos y personas realmente } \\
\text { interesantes al margen de los sitios que conoce todo el mundo }\end{array}$ &, 525 &, 303 &,- 164 &,- 012 \\
\hline $\begin{array}{l}\text { Creo que gracias a las redes sociales puedes llegar a ser muy } \\
\text { popular y tener mucho éxito }\end{array}$ &, 507 &, 069 &, 068 &,- 234 \\
\hline $\begin{array}{l}\text { Hoy en día no te puedes fiar mucho de los contenidos de las } \\
\text { redes sociales porque la gente se aprovecha del anonimato y } \\
\text { muchos contenidos no son ciertos }\end{array}$ &,- 410 &, 561 &, 046 &, 243 \\
\hline $\begin{array}{l}\text { Suelo encontrar contenidos casualmente pasando de un enlace } \\
\text { a otro }\end{array}$ &, 362 &, 619 &,- 184 &, 158 \\
\hline $\begin{array}{l}\text { Cuando encuentro una página, blog o grupo que me interesan } \\
\text { sus contenidos lo agrego a mi perfil, al RSS, o a mis favoritos } \\
\text { para seguirlo con }\end{array}$ &, 067 &, 549 &,- 248 &,- 135 \\
\hline $\begin{array}{l}\text { Suelo confiar bastante en los comentarios que dejan otros } \\
\text { usuarios en Internet cuando voy a hacer una compra, bajar un } \\
\text { contenido o busco otro tipo de información }\end{array}$ &, 031 &, 531 &,- 030 &, 058 \\
\hline
\end{tabular}




\begin{tabular}{|l|c|c|c|c|}
\hline & \multicolumn{3}{|c|}{ Dimensiones } \\
\hline & $\mathbf{1}$ & $\mathbf{2}$ & $\mathbf{3}$ & $\mathbf{4}$ \\
\hline $\begin{array}{l}\text { Para mi la red social es una oportunidad para enterarte de mu- } \\
\text { chas cosas y entrar en contacto con otras personas que tienen } \\
\text { tus mismos intereses }\end{array}$ &, 332 &, 492 &, 214 &,- 282 \\
\hline $\begin{array}{l}\text { Para mi entrar en Internet y buscar contenidos es una forma de } \\
\text { entretenimiento }\end{array}$ &, 334 &, 434 &, 002 &, 061 \\
\hline $\begin{array}{l}\text { Cuando hago búsquedas en la red con google, yahoo! o similar } \\
\text { soy bastante directa/o voy directamente a lo que me interesa y } \\
\text { no suelo explorar sólo por curiosear }\end{array}$ &,- 066 &,- 100 &, 655 &,- 025 \\
\hline $\begin{array}{l}\text { Tengo una rutina muy definida cuando navego en Internet y no } \\
\text { suelo entrar en sitios que no conozco o me han recomendado }\end{array}$ &,- 089 &, 016 &, 646 &, 106 \\
\hline $\begin{array}{l}\text { Sólo me interesa Internet para estar al día y ver la actividad de } \\
\text { mis contactos cercanos }\end{array}$ &, 041 &,- 098 &, 639 &,- 097 \\
\hline $\begin{array}{l}\text { Soy bastante escéptico en participar en eventos u otras accio- } \\
\text { nes en las redes sociales que puedan dejar visible mi perfil a } \\
\text { desconocidos }\end{array}$ &,- 104 &, 045 &, 062 &, 730 \\
\hline $\begin{array}{l}\text { Pienso que en Internet hay contenidos que valen la pena pero } \\
\text { también hay mucha “basura” }\end{array}$ &, 059 &, 027 &, 126 &, 652 \\
\hline $\begin{array}{l}\text { Soy bastante crítico con los contenidos que veo en la redes } \\
\text { sociales, para mi gusto hay muchas chorradas que no tienen } \\
\text { credibilidad }\end{array}$ &,- 058 &, 026 &,- 153 &, 413 \\
\hline Proporción de varianza explicada & $12,1 \%$ & $11,6 \%$ & $9,4 \%$ & $9,4 \%$ \\
\hline
\end{tabular}

Fuente: Elaboración propia

\subsection{Relación entre perfil de usuario, tiempo de consumo e impli- cación en actividades publicitarias}

A continuación, se pone a prueba el segundo objetivo del estudio, que es la relación entre las variables que reflejan el estilo de navegación de los jóvenes usuarios y su comportamiento en la red. Para ello, se lleva a cabo un análisis de correlación en el que se han incluido los cuatro perfiles recogidos en el análisis factorial ${ }^{8}$, el grado de implicación con la publicidad en Internet y el tiempo de consumo en Internet durante la semana (lunes a viernes) y durante el fin de semana (sábado y domingo).

En el cuadro $n^{0} 3$ se muestran los coeficientes de correlación de Pearson obtenidos para cada par de variables así como su significación estadística. Según se puede 
apreciar en los resultados los perfiles identificados tienen un patrón específico en su relación con las dos variables comportamentales. Por una parte, el perfil confiado y sociable presenta una relación de signo positivo y estadísticamente significativa con la implicación en actividades publicitarias y comerciales $\left(\mathrm{r}=0,193^{* *}\right.$; sig. $=0,001)$. Según el sentido de la relación cuanto más se confían los jóvenes en la red y la perciben como vehículo de socialización, mayor es su implicación en actividades publicitarias. De igual modo, encontramos que las personas que se identifican con este perfil tienden a pasar más tiempo conectadas a Internet durante los días laborables $\left(r=0,178^{* *}\right.$, sig. $\left.=0,003\right)$ y durante los fines de semana $\left(r=0,130^{*}\right.$, sig. $=0,029)$. El grado de asociación en ambos casos es de magnitud moderada, no obstante, la relación es estadísticamente significativa. Si bien, se aprecia que esa relación es más fuerte con el tiempo de conexión durante la semana que durante el fin de semana. En contraste, los individuos que se identifican con el perfil escéptico hacia las redes sociales no han mostrado tener relación con la implicación publicitaria $(\mathrm{r}=0,026)$, ni con el tiempo de conexión a Internet semanal $(\mathrm{r}=$ $0,050)$, o el tiempo de conexión durante el fin de semana $(r=-0,037)$. La ausencia de relación implica la independencia de ambos sucesos, por lo tanto, podemos afirmar que entre los usuarios más escépticos encontramos a jóvenes más implicados en actividades publicitarias y jóvenes menos implicados en este tipo de acciones. Lo mismo puede decirse del tiempo de conexión a Internet.

El estilo de navegación exploratorio también muestra una relación de signo positivo con la implicación de los jóvenes en actividades publicitarias y comerciales ( $\mathrm{r}$ $=0,290^{* *}$; sig. $\left.=0,000\right)$. La relación es estadísticamente significativa y la magnitud de la relación, aun siendo moderada, presenta un grado de asociación con el comportamiento en la red que el perfil actitudinal de confianza en las redes. De igual modo, el estilo exploratorio se asocia positivamente con el tiempo de conexión a Internet de lunes a viernes $\left(\mathrm{r}=0,190^{* *}\right.$, sig. $\left.=0,001\right)$ y con el tiempo de conexión durante el fin de semana $\left(r=0,141^{*}\right.$, sig. $\left.=0,020\right)$. En ambos casos, el perfil exploratorio muestra un grado de asociación más débil con el tiempo de conexión que con la participación en las actividades de la red. Por último, el estilo de navegación focalizado no muestra una relación estadísticamente significativa con la implicación en actividades publicitarias $(r=-0,014)$, ni con el tiempo de conexión a Internet de lunes a viernes $(r=0,002)$, ni con el tiempo de conexión durante el

ICONO14 | Año 2014 Volumen $12 N^{\circ} 1$ | ISSN: 1697-8293 | DOI: ri14.v12i1.208 
fin de semana $(r=0,099)$. Como ocurriera con los escépticos en las redes sociales, el tener un estilo de navegación focalizado no influye en el tiempo de navegación, ni en el grado de participación en las propuestas de los anunciantes en el medio. En el cuadro $n^{0} 3$ también se puede apreciar la ausencia de relación entre la participación en actividades publicitarias y el tiempo de conexión a Internet $(\mathrm{r}=0,022$ y $\mathrm{r}=0,099$ para la conexión durante la semana y el fin de semana respectivamente).

\section{Cuadro $n^{\circ}$ 3. Análisis de correlación de Pearson entre el tiempo de consumo, estilo de navegación, actitud hacia internet y la implicación en actividades publicitarias}

$\begin{array}{lccc} & \begin{array}{c}\text { Implicación } \\ \text { publicitaria } \\ \text { Perfil sociable y confiado }\end{array} & \begin{array}{c}\text { Tiempo conectado a } \\ \text { Internet (L a V) }\end{array} & \begin{array}{c}\text { Tiempo conectado a } \\ \text { Internet (S y D) }\end{array} \\ \text { Perfil escéptico redes sociales } & 0,193 * * & 0,178^{* *} & 0,130 * \\ \text { Estilo nav egación exploratorio } & 0,290 * * & -0,050 & -0,037 \\ \text { Estilo de navegación focalizado } & -0,014 & 0,190 * * & 0,141 * \\ \text { Implicación publicita ria } & 1 & 0,002 & -0,055 \\ \text { Tiempo conectado a Intern et (L a } & & 0,022 & 0,099 \\ \text { V) } & & 1 & 0,622 * *\end{array}$

${ }^{*}$ La correlación es significativa al nivel 0,01 * La correlación es significativa al nivel 0,05

Fuente: Elaboración propia

\section{Conclusiones y Discusión}

En el estudio se ha comprobado que los jóvenes y adolescentes muestran patrones 0 perfiles diferenciados en el consumo de medios interactivos. El hecho de que los menores pasen mucho tiempo en la red no implica que todos se estén enfrentando del mismo modo al medio interactivo.

La escala actitudinal y de estilo de navegación propuestos en este trabajo se han puesto a prueba empíricamente, corroborando la diferenciación de cuatro perfiles que giran en torno a los usos diferenciados en la red. En este sentido se han completado las propuestas de Shiffman y col. (2003) y de Assael (2005) sobre la categorización de los usuarios en el medio interactivo que nos ayudan a comprender mejor su comportamiento 
en le medio. Tal como se ha evidenciado en el trabajo, los dos primeros perfiles tienen un componente actitudinal, consideramos que la confianza versus escepticismo en la red son cuestiones donde el aspecto cognitivo es bastante relevante. Los argumentos 0 creencias sobre las que se fundamenta su comportamiento precavido versus confiado en las redes dan coherencia a su forma de enfrentarse al medio interactivo. Posiblemente estas actitudes se hayan formado en torno a su experiencia en el medio interactivo y pueden ser modificadas. Este resultado tiene importantes implicaciones de cara a concienciar a los menores sobre los riesgos en Internet y la alfabetización digital. Podemos concienciar a los menores de la importancia de la seguridad en el medio, el tener una actitud preventiva y crítica frente a un exceso de confianza.

Por otra parte, el estilo de navegación refleja en mayor medida un aspecto más cercano a las variables de personalidad. Algunos jóvenes se identifican más con un estilo explorador a la hora de enfrentarse al medio y lo perciben como una fuente de información y de estimulación. En cambio, otros se identifican más con un estilo más focalizado, guiado por sus propios objetivos $\mathrm{y}$, en consecuencia, es menos susceptible de dejarse llevar por los estímulos externos. Los resultados de los perfiles han sido positivos, y se ha comprobado su relación con variables comportamentales, lo que ratifica su validez externa. No obstante, las escalas no se habían utilizado con anterioridad, de modo que estos resultados deberán corroborase con posteriores trabajos.

Otro aspecto relevante del trabajo lo encontramos en la alta participación en actividades publicitarias, aunque no tenemos datos de referencia para su interpretación, lo cierto es que se corrobora la disposición positiva de los menores a interactuar con las marcas y anunciantes, aspecto contemplado en los informes de la Consultora The Cocktail Analysis. Una cuestión que lleva a confirmar el papel activo de los receptores en la comunicación en Internet y que de alguna forma contradice la afirmación de que la publicidad en este medio se percibe de forma negativa y provoca rechazo, como se plantea en trabajos anteriores (Frutos y Sánchez-Valle, 2011).

Posiblemente la mayor aportación de este trabajo sea haber puesto en relación los perfiles de jóvenes y adolescentes con el comportamiento en la red. Por una parte, se pone de manifiesto que la actitud de confianza hacia el entorno interactivo y el estilo exploratorio influyen en el consumo de medio. Los más confiados están más tiempo 
expuestos a los contenidos del medio interactivo y además, se pone más de manifiesto durante la semana que el fin de semana. Este patrón se repite para el estilo de navegación exploratorio, que también se conectan más y la influencia es mayor durante la semana que en el fin de semana. Igualmente, este patrón de relación se mantiene para la participación en actividades vinculadas a la publicidad o a las marcas. Podríamos pensar que el tiempo de conexión podría ser una explicación para que los menores participen más en este tipo de iniciativas, esto es, que hubiera relación entre el tiempo de conexión y la implicación publicitaria. Sin embargo, el estadístico que mide la asociación entre ambos sucesos demuestra que son independientes. Esto tiene importante implicación práctica de cara a la educación, al poner de relevancia que es más importante la actitud y el estilo de navegación de los menores, que estrategias de control, como por ejemplo, limitar el tiempo de conexión en Internet. Aunque en este trabajo no se medido variables como rendimiento escolar, sería muy interesante ver si también alguno de los perfiles se relacionan con los resultados académicos.

En cuanto a las limitaciones del estudio se pueden señalar varias. Este estudio aborda sólo un pequeño aspecto del uso que los adolescentes realizan de la red, sería conveniente ampliar el objeto de análisis a otros comportamientos cercanos que posiblemente nos daría una visión más global de las implicaciones del medio y otras variables personales, sociales o de otro tipo que influyen en el comportamiento. Por otra parte, sería interesante complementarlo con otras metodologías, puesto que la correlacional tiene sus limitaciones a la hora de establecer relaciones de influencia.

A pesar de las limitaciones del estudio, se pueden derivar varias implicaciones prácticas a partir de los resultados obtenidos ya mencionadas, que podrían quedar resumidas en una sola la importancia del componente actitudinal en la alfabetización digital o educación en el medio digital. A lo largo del trabajo se pondera la importancia que tiene la actitud hacia el medio en el comportamiento. Las consecuencias prácticas son evidentes, desde los educadores, padres tutores se puede realizar una labor de concienciación para fomentar una actitud crítica hacia el medio. Padres y educadores pueden ayudar a los más jóvenes a tomar conciencia de cuestiones como la privacidad, el escepticismo hacia los contenidos, la importancia de utilizar la herramienta para los propios intereses, en lugar del camino inverso. Cuestiones que están por encima de la esfera tecnológica pero que posiblemente sean una aportación muy valiosa en el aprovechamiento de la tecnología. 


\section{Notas}

[1] Los datos del Estudio general de medios se refieren al día de ayer, por ello la penetración del medio arroja datos inferiores a las otras fuentes.

[2] EUROBAROMETER. Disponible en http://ec.europa.eu/public_opinion/archives/ebs/ebs_335_ en.pdf (consultado 20 de enero de 2012).

[3] La navegación libre hace referencia al estilo de actuación en las situaciones en las que el usuario no está delimitado por el tiempo o el tipo de acción que tiene que llevar a cabo en la red. Las preguntas de este apartado iban encabezadas por la siguiente cuestión: Cuando tengo tiempo para navegar...

[4] La variable se ha calculado a partir de la suma de la participación en las actividades publicitarias y comer-ciales de Internet. El valor máximo de esta escala es de 10 puntos.

[5] Los dispositivos Smartphone tiene una penetración en España muy alta. En el momento en el que se llevó a cabo la recogida de datos, el uso de internet en el móvil contaba con un índice de penetración que roza el 50\%, según la revista Marketing directo.com. En http://www.marketingdirecto.com/actualidad/digital/espana-lider-en-el-uso-de-internet-movil/ (consultado 8 de septiembre de 2011).

[6] En el cuestionario se describía brevemente este tipos de juegos, que aparecen en ventanas emergentes en la navegación por Internet, no requieren el registro, ni la descarga por parte del usuario y normalmente detrás hay un servicio de tarificación por SMS o un anuncio publicitario de algún anunciante. Este tipo de acciones han aparecido frecuentemente bajo modalidades de test de inteligencia, test de atención, de rapidez o similares, pero cons-tantemente aparecen nuevas versiones.

[7] Aunque no tenemos una estadística oficial para contrastar este grado de participación, consideramos que está por encima de los datos manejados por el sector según un reciente estudio publicado por la revista marketing news publicado en diciembre de 2011, que sitúa la ratio de conversión de fans en el $26 \%$.

http://www.marketingnews.es/tendencias/noticia/1061732029005/26-usuarios-espanolesredes-sociales.1.html

[8] Para obtener las puntuaciones en los perfiles se ha seguido el método de regresión a partir de los pesos en los factores. Las puntuaciones factoriales están normalizadas de modo que la puntuación media es cero y la desviación típica es igual a 1. Como el método de extracción de los factores ha seguido el método oblimin las dimensiones son independien-tes, por lo tanto la correlación entre las puntuacio-nes factoriales es cero. 


\section{Referencias}

AIMC (2012). Estudio General de Medios. $3^{\circ}$ ola: Febrero a Noviembre de 2012. Disponible en: http://www.aimc.es/-Datos-EGM-Resumen-General-.html. Consultado el 26 de 02 de 2013.

Assael, H. (2005). A Demographic and Psychographic Profile of Heavy Internet Users and Users by Type of Internet Usage. Journal of Advertising Research, March, 93-123.

Bringué, J. y Sádaba, C., (2011). Menores y redes sociales. Foro generaciones interactivas. Madrid: Fundación Telefónica, 2011.

Dahlén, M. Rash, A y Rosegren, S. (2003): "Love at first site? A study of website Advertising effectiveness", Journal of Advertising Research, 43, 25-33.

Frutos Torres, B. (2010). "Alternativas a las medidas de eficacia publicitaria para un nuevo paradigma de comunicación". En Perlado M. y Jimenez, C. Escenario actual de investigación en comunicación: objetivos, métodos y desafíos. Pp. 411-421, EDIP0, Madrid, 2010.

Frutos Torres, B. y Gutiérrez Zotes, P. (2005): “Respuestas cognitivas de los usuarios a los contenidos publicitarios en "rich media". X Congreso Internet, Telecomunicaciones y Sociedad de la Información. Mundo Internet, Madrid.

Frutos Torres, B. y Sánchez Valle, M. (2006). “Productos funcionales versus productos expresivos y de alta implicación versus baja implicación en Internet". Comunicación y pluralismo, $\mathrm{N}^{\circ} 1$, pp. 35-78.

Frutos Torres, B. y Sánchez Valle, M. (2011). "Aproximación empírica a la percepción de la publicidad on display en Internet". Sphera Pública. Revista de Ciencias Sociales y de la Comunicación, $\mathrm{n}^{0} 11$. Pp. 217-235.

Frutos Torres, B. y Vázquez Barrio, T. (2012). “Adolescentes y jóvenes en el entorno digital: análisis de su discurso sobre usos, percepción de riesgo y mecanismos de protección". Doxa. Revista Interdisciplinar de estudios de Comunicación y Ciencias Sociales, $n^{0}$ 15, Pp 57-59.

García Jiménez, A.; López de Ayala, M. C. y Gaona Pisonero, C. (2011). La investigación sobre los usos y los riesgos de los menores en el ciberespacio. Análisis metodológico. Doxa comunicación, $\mathrm{n}^{0}$ 13, pp. 14 - 41. Garitaonandia, C.; Garmendia, M. (2009): Cómo usan Internet los jóvenes: hábitos, riesgos y control parental. Universidad del País Vasco/Euskal Herriko

DOI: ri14.v12i1.208 | ISSN: 1697-8293 | Año 2014 Volumen 12 Nº 1 | ICONO14 
Unibertsitatea, Bilbao: EU Kids Online.

Garmendia, M.; Garitaonandia, C.; Martínez, G.; Casado, M. A. (2011): Riesgos

y seguridad en internet: Los menores españoles en el contexto europeo.

Universidad del País Vasco/Euskal Herriko Unibertsitatea, Bilbao: EU Kids Online.

Hasebrink, U.; Livingstone, S.; Haddon, L. (2008): Comparing children's online opportunities and risks across Europe: Crossnational comparisons for EU Kids Online. London: EU Kids Online. Disponible en http: www.eukidsonline.net. Consultado el 7 de enero de 2010.

INE (2011). Encuesta sobre Equipamiento y Uso de Tecnologías de Información y Comunicación en los Hogares. Año 2011. Disponible en: http:/www.infoadex. es. Consultado el 20 de Octubre de 2011.

INFOADEX (2011). Estudio Infoadex de la inversión publicitaria en España 2011.

Disponible en http:/www.infoadex.es. Consultado el 10 de 0ctubre de 2011. Livingstone, S. (2010): “Youthful participation: what have we learned, what shall we ask next?", First Annual Digital Media and Learning Conference: Diversifying Participation, 18-20 February 2010, University of California, San Diego, La Jolla, California. (Unpublished). Disponible en: http://eprints.lse. ac.uk/27219/. Consultado 10 de junio de 2011.

Livingstone, S. y Haddon, L., (2009). Kids Online: Opportunities and Risks for Children. The Policy, Bristol, 2009.

Monge, S.y Olabarri, M. E., (2011). “Los alumnos de la UPV/EHU frente a Tuenti y Facebook: usos y percepciones" en Revista Latina de Comunicación Social, 66, pp. 79-100. Disponible en: http://www.revistalatinacs.org/11/art/925_ UPV/04_Monge.html. Consultado el 2 de marzo 2011.

Muylle, S. Moenaert, R. \& Despontin, M. (1999). A grounded theory of World Wide Web search behaviour. Journal of Marketing Communications, 5, 143155.

Phang, A. y Schaefer, D. J. (2009). “Is Ignorance Bliss? Assesing Singaporean Media Literacy Awarness in the Era of Globalization". Journalism and Mass Communication Educator. Vol. 64 (2009), no 2, p. 156-172.

Rubio, A. (2010). Generación digital: patrones de consumo de Internet, cultura juvenil y cambio social. Revista de estudios de Juventud, $\mathrm{n}^{0}$ 88, pp. 201221. 
Sánchez Burón, A.; Fernández Martín, M. P. (2010): Informe Generación 2.0. Hábitos de los adolescentes en el uso de las redes sociales. Estudio comparativo entre Comunidades Autónomas, Madrid: Universidad Camilo José Cela. Disponible en: http://estaticos.elmundo.es/documentos/2010/07/06/ Generacion20.pdf. Consultado en 10 de junio de 2011. Sánchez-Valle, M. y Frutos, B. (2010): “La investigación de la publicidad interactiva dirigida a niños y adolescentes". En Perlado M. y Jimenez, C. Escenario actual de investigación en comunicación: Objetivos, Métodos y Desafios. pp. 330-341, Edipo, Madrid.

Sánchez-Martínez, M. y Otero, A., (2010). “Usos de Internet y factores asociados en adolescentes de la Comunidad de Madrid" en Atención Primaria, $\mathrm{n}^{0}$ 42(2), pp. 79-85.

Schiffman, L. G., Sherman, E., \& Long, M. M. (2003). Toward a better understanding of the interplay of personal values and the internet. Psychology \& Marketing, 20(2), 169-186. Retrieved from http://search. proquest.com/docview/227679592?accountid=14778

The Cocktail Analysis (2011). Informe de resultados Observatorio Redes Sociales. Recuperado en: http://www.tcanalysis.com/uploads/2011/02/0bservatorioRedesSociales2011.pdf

Yang K. C. C. (2004). Effects of Consumer Motives on Search Behavior Using Internet Advertising. CyberPsychology \& Behavior. Vol. 4, nº 4, 430-442.

\section{Agradecimientos}

Las autoras de este trabajo queremos agradecer a la Universidad CEU San Pablo por la financiación recibida para realizar este proyecto con a través de sus ayuda a la investigación (referencia USP-PI-5/09). También reconocemos públicamente la colaboración de dos instituciones privadas: Nielsen/ Net-Rating Spain y AdHoc Investigación de Mercados, por su colaboración desinteresada en el proyecto y la aportación de datos que han facilitado el curso de la investigación. Gracias a los centros de enseñanza secundaria y a todas las personas que colaboraron en la recogida de datos, $\mathrm{y}$, por último, nuestro sincero agradecimiento a los revisores anónimos del trabajo por su tiempo y sus valiosos comentarios.

DOI: ri14.v12i1.208 | ISSN: 1697-8293 | Año 2014 Volumen 12 Nº 1 | ICONO14 
397 | Belinda de Frutos Torres, María Sánchez Valle, Tamara Vázquez Barrio

Cita de este artículo

(2014) Perfiles de adolescentes on line y su comportamiento en el medio interactivo [en línea] 31 de enero de 2014, No 1. pp. 374-397. Recuperado (Fecha de acceso), de http://www.icono14.net

ICONO14 | Año 2014 Volumen $12 \mathrm{~N}^{\circ} 1$ | ISSN: 1697-8293 | DOI: ri14.v12i1.208 